\title{
Problems of the conventional BL model as applied to super/hy- personic turbulent boundary layers and its improvements
}

\author{
DONG Ming ${ }^{1,2^{*}} \&$ LI XinLiang ${ }^{3}$ \\ ${ }^{1}$ Department of Mechanics, Tianjin University, Tianjin 300072, China; \\ ${ }^{2}$ Tianjin Key Laboratory of Modern Engineering Mechanics, Tianjin 300072, China; \\ ${ }^{3}$ Key laboratory of High Temperature Gas Dynamics, Institute of Mechanics, Chinese Academy of Sciences, Beijing 100190, China
}

Received January 31, 2011; accepted June 29, 2011; published online August 30, 2011

\begin{abstract}
Turbulence modeling has played important roles in solving engineering problems. However, with the development of aerospace technology, turbulence modeling faces new challenges. How to further improve turbulence modeling for super/hypersonic flows is an urgent problem. Through analyzing a set of data resulting from DNS and experiments, it is found that some most popular models suffer from essential flaws, and can be hardly improved following the traditional mode of thinking. On the contrary, the BL model, which is one of the simplest and widely-used models, can be further improved. In this paper, through analyzing results from DNS data, the main cause of the inaccuracy in applying the BL model to supersonic and hypersonic turbulent boundary layers is found to have resulted from the mismatch between the location of the matching point of the inner and outer layers of the BL model determined by the conventional way and those given by DNS. Improvement on this point, as well as other improvements is proposed. Its effectiveness is verified through the comparison with DNS results.
\end{abstract}

turbulence modeling, supersonic, hypersonic, turbulent boundary layer, DNS, BL model

PACS: 47.27.E-, 47.27.ek, 47.27.nb

Currently, turbulence modeling is the only tool for engineering computation of turbulent flows. Of the many different turbulence models, the most popular ones are those using the concept of eddy viscosity [1-5]. Each model has quite a number of adjustable parameters to be determined by comparing them with experimental results. The adjustable parameters are usually determined for incompressible flows, and do not bear universality. Hence when the model is applied to super/hypersonic turbulent flows, the results are often unsatisfactory. How to make them fit the requirement for super/hypersonic flows is an unsolved problem [6].

The $k-\varepsilon$ and $k-\omega$ models are the most popular two-equation models using the concept of eddy viscosity. Through the application and constant modification for years, for incompressible turbulent flows, they can provide reasonable

*Corresponding author (email: dongming@tju.edu.cn) results, at least for simple incompressible flows. If the model can be further improved, the question for many turbulence researchers is how. On page 134 of ref. [7], Wilcox provided the results for an incompressible turbulent channel flow, computed by the $k-\omega$ model. It shows that the distribution of the turbulent kinetic energy $k$ is more or less correct qualitatively compared to experiment results, while the distribution of the dissipation $\varepsilon$ is incorrect even qualitatively in the near wall region. However, the model can still yield reasonable distribution of the eddy viscosity coefficient in this case, which certainly contributed to adjustable parameters rather than the correctness of the concept involved. On the other hand, Figure 1 shows the distribution of the eddy viscosity coefficient (the unfilled circles) for Mach 2.25 turbulent boundary layer on a flat plate, derived by Li et al. [8] from their DNS. Shown also in the figure is the eddy viscosity coefficient computed by the standard 


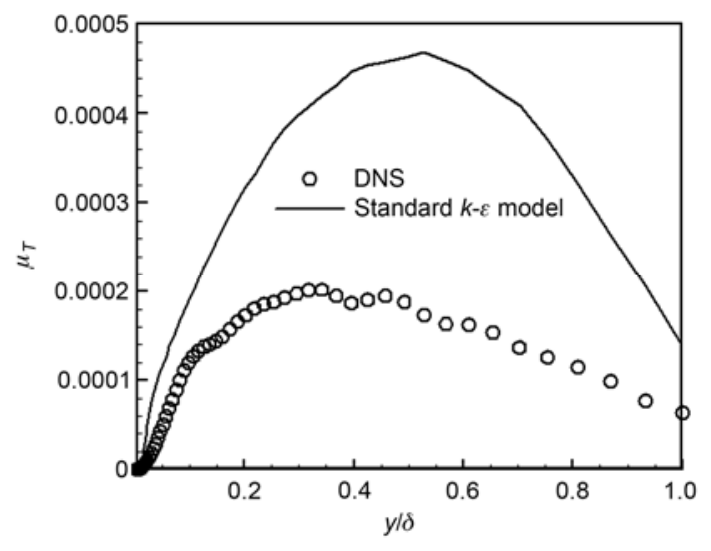

Figure 1 Eddy viscosity coefficient.

formula of $k-\varepsilon$ model (the solid line), but instead with $k$ and $\varepsilon$ obtained from their respective equations as in the model, given directly by DNS data. What astonished us is that they are different from each other not only quantitatively, as one would expect, but also qualitatively. This suggests that the original idea of the model that the eddy viscosity coefficient $\mu_{T}$ is proportional to $k^{2} / \varepsilon$, based purely on dimensional analysis, is incorrect. The reason might be that in different regions of the boundary layer, the dynamics might be different. In the wall region, there exist coherent structures, while in the outer region, the turbulent structures are weaker and with different scales. Therefore, it is hopeless to further improve the model rationally under the original formulation. The only practical way is by adding more adjustable parameters to the model. For example, let $\mu_{T}$ be proportional to $k^{2} / \varepsilon$, but the coefficient of proportion is not a constant but a function of $y$, the normal coordinate.

On the contrary, although those simple models, such as BL model [1], do not appear to be so sophisticated as the two equation model, they can be further improved by simple reasoning.

The basic idea of the BL model is that the boundary layer can be divided into two regions, namely, the inner layer and the outer layer, and the distribution of the eddy viscosity coefficient in these two regions can be obtained respectively by simple reasoning. Figure 2 is the near-wall-law of the mean stream-wise velocity of a turbulent boundary layer, cited from page 45 of ref. [7], and clearly the velocity profile has three distinct regions in the normal direction. The Sublayer and the Log Layer belong to the inner layer, for which the eddy viscosity coefficient is given by the mixing length theory in BL model. The Defect Layer belongs to the outer layer, for which the eddy viscosity is given by an empirical wake flow function in the model. Point $\mathrm{C}$ in the figure is the upper bound of the Log Layer, which is the upper limit of the region, in which the mixing length theory applies; while point $\mathrm{D}$ is the intersection of the Sublayer and the Log Layer. The idea of dividing the boundary layer into two regions has clear physical meaning, as stated above, i.e.

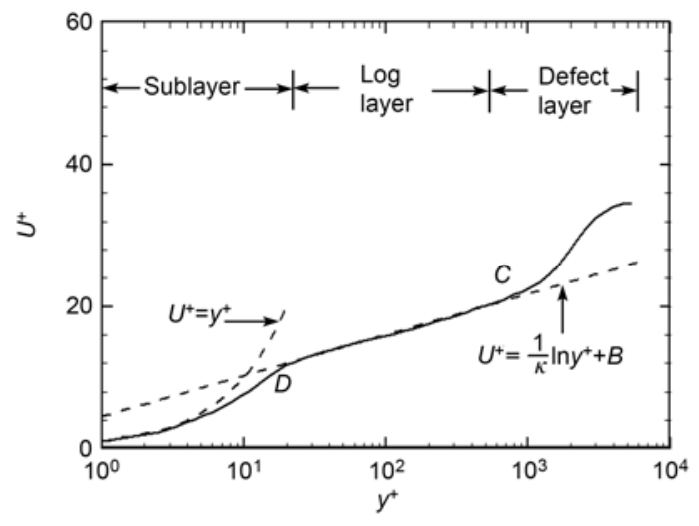

Figure 2 Near-wall-law of the mean profile.

they have different dynamics. For incompressible turbulent boundary layers, if normalized by the viscous length, the normal coordinate of the dividing point $\mathrm{C}$ of the two regions is more or less a constant, nearly 100 , irrespective of what is the Reynolds number of the boundary layer. For the compressible turbulent boundary layer, this is also true, at least within a certain range of Reynolds and Mach numbers, as confirmed by our DNS. While on the other hand, the total thickness of the boundary layer, also measured by viscous length, depends on both the Mach number and the Reynolds number.

Though the model is simple and widely used, the accuracy of the result from the model is not high enough. In Baldwin and Lomax's paper [1], many examples of application, including different types of turbulent flows, were listed. However, examining their first example carefully, namely, the turbulent boundary layer on flat plates with Mach 1.5 and 2.85 , one would find that the resulting skin friction coefficient and the momentum thickness of the boundary layer are not accurate enough, differing from the experimental data up to about $20 \%$.

Based on the DNS data, Dong and Zhou [9] examined the accuracy of the BL model as applied to super/hypersonic turbulent boundary layers. It was found that the aerothermal quantities at the wall and the mean flow profiles yielded by applying the model did have certain discrepancies in comparison with the DNS results. Accordingly, they proposed a modification on the turbulent Prandtl number, which yielded positive result on improving the accuracy of aerothermal quantities [10]. But in that paper, the improvement on eddy viscosity coefficient was not considered, and consequently, the mean flow profiles still had obvious differences compared with the DNS results.

The key issue found affecting the accuracy of the result in applying the BL model to super/hypersonic turbulent boundary layer is the location of point $\mathrm{C}$, dividing the inner layer and the outer layer, as shown in figure 2. Although the mixing length theory is only a phenomenological theory, it has stood many tests. It can be well applied to compute the 
inner layer of wall bounded turbulent flows, including the whole Log Layer. Based on experiments, Escudier [11] pointed that the normal coordinate of point $\mathrm{C}$ should not exceed $0.09 \delta$, where $\delta$ is the nominal boundary layer thickness. However, recently, more DNS for super/hypersonic turbulent boundary layers studies found the above conclusion untrue for super/hypersonic turbulent boundary layers. Thus, the first task of this paper is to offer a method to determine the location of point $\mathrm{C}$, dividing the inner and outer layers.

Another important issue is that for super/hypersonic turbulent boundary layers, unlike those incompressible flows, the wall temperature condition also plays an important role, that is, even the Mach number and Reynolds number being the same, the mean flow profile can be significantly different under different wall temperature conditions. Duan et al. [12] performed DNS for turbulent boundary layers with the same Mach number under 5 different wall temperature conditions, from which one can find that the distance from the wall to point $\mathrm{D}$ in Figure 2 depends on the wall temperature condition. The lower the wall temperature is, the larger the distance is. This factor will also be considered for the improvement of BL model.

Furthermore, in the case of cold wall, the turbulent Prandtl number should be modified in the near wall region, as suggested in ref. [10], but a minor change will be suggested.

\section{The location of the dividing point of inner and outer layers in turbulent boundary layers}

Based on the DNS data of turbulent boundary layer in refs. $[10,12-18]$, the normal distance of the dividing point of inner and outer layers under different Reynolds number and Mach number are listed in table 1, including turbulent boundary layers on both flat plates and cones. The Mach number ranges from 2.5 to 6.0, and the Reynolds number $R e_{\theta}$, based on momentum thickness, are all several thousands except Case 14. $T_{\mathrm{w}} / T_{\mathrm{aw}}$ is the ratio of the wall temperature and the adiabatic temperature. If $T_{\mathrm{w}} / T_{\mathrm{aw}}=1$, it is adiabatic wall; if $T_{\mathrm{w}} / T_{\mathrm{aw}}>1$, it is hot wall; otherwise it is cold wall. $y_{c} / y_{\delta}$ is the ratio of the distance of the dividing point from the wall and the nominal boundary layer thickness.

It has been mentioned above that the inner layer of a turbulent boundary layer, in which mixing length theory works, is the region where large scale coherent structures exist. Tsujimoto and Miyake [19] found, through DNS of an incompressible turbulent channel flow, that if the computational region was limited to a region close to the wall, and the normal velocity at the upper boundary was set to be zero, implying that there is no momentum exchange between that wall region and the outer region, and the characteristics of turbulence, including the characteristics of the coherent structures remain unchanged even when the distance of the upper boundary from the wall is only 100 wall unit. This is just the extent of the inner layer of a turbulent boundary layer. The filled circles in Figure 3 give the relationship between $y_{c}^{+}$and $\operatorname{Re}_{\theta}$ for Cases 10-14, corresponding to boundary layers on a flat plate with Mach 6 and adiabatic wall. It can be seen that although the Reynolds number varies greatly, hence also the boundary layer thickness $y_{\delta}^{+}$, $y_{c}^{+}$clustered around the value 100.

Generally speaking, the boundary layer thickness, scaled by viscous length, increases as the Reynolds number increases, and decreases as the Mach number increases, as shown by the filled square symbols in Figure 3.

Table 1 The location of the intersection in different cases

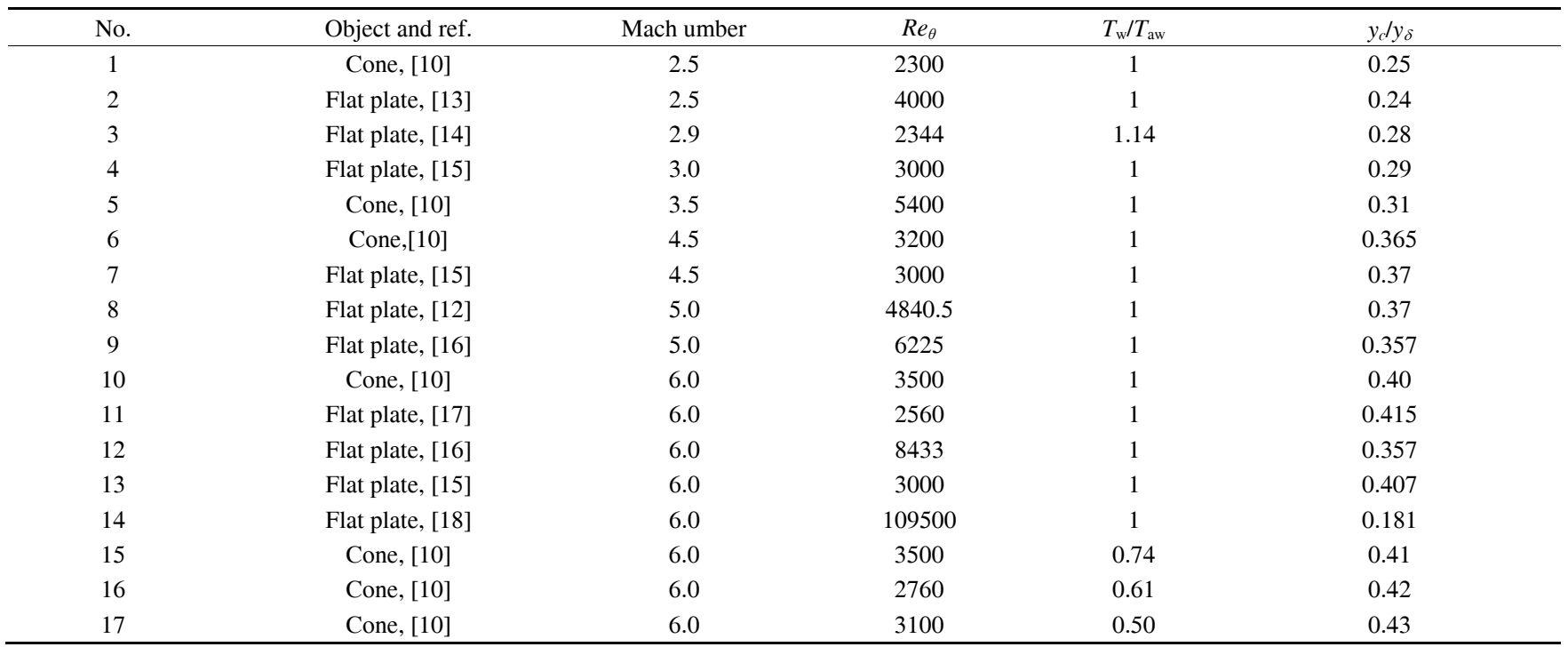


Based on all the data in table 1 , the ratio $y_{c} / y_{\delta}$ as a function of $\operatorname{Re}_{\theta}$ and $M a$ can be expressed as:

$$
\begin{aligned}
y_{c} / y_{\delta}= & (-0.65 \exp (-0.4 M)+0.32) \exp \left(-0.00005 R e_{\theta}\right) \\
& +0.18 .
\end{aligned}
$$

The curved surface, determined by the equation in space M- $R e_{\theta}-y_{c} / y_{\delta}$, is shown in Figure 4. The filled square symbols in the figure represent the data in Table 1. As can be seen, they all stand almost exactly on the curved surface. Therefore, in turbulence modeling, one can use eq. (1) to determine in advance the dividing point of the inner layer and the outer layer.

\section{Method for improving the model}

Based on the discussions above, the BL model should be modified in 3 aspects, when applied to super/hypersonic turbulent boundary layers.

\subsection{Modification I: on the location of the dividing point of inner and outer layers}

In BL model, based on the mixing length theory, the eddy

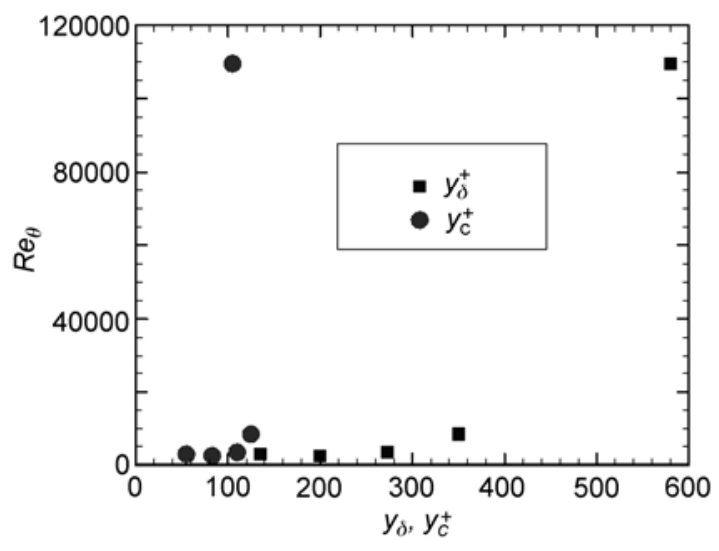

Figure 3 Distribution of $y_{c}^{+}$and $y_{\delta}^{+}$.

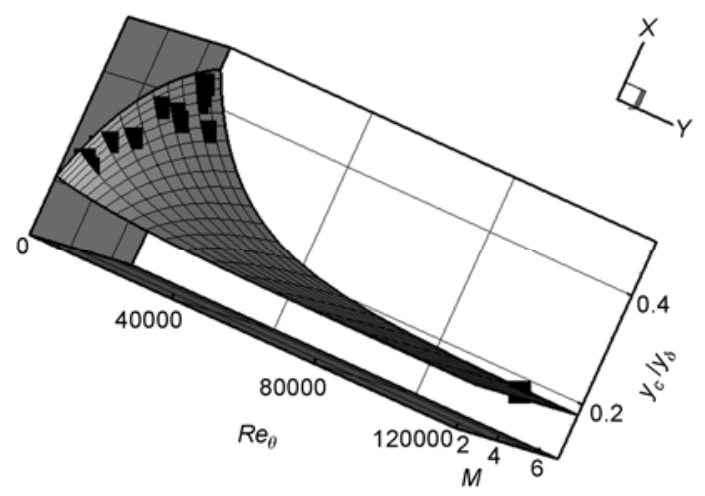

Figure 4 Curved surface by eq. (1). viscosity coefficient in the inner layer is expressed as:

$$
\mu_{T}=\rho l_{\mathrm{mix}}^{2} \frac{\partial U}{\partial y},
$$

where $\rho, U$ are the mean density and mean stream-wise velocity, respectively, $y$ the normal coordinate, and $l_{\text {mix }}$ the mixing length, which is expressed as:

$$
l_{\text {mix }}=\kappa y\left[1-\exp \left(-y^{+} / A^{+}\right)\right],
$$

where $\kappa$ is Karman constant, usually set to be $0.4 ; y^{+}$the viscous length, and $A^{+}=26$.

For the outer layer, the eddy viscosity coefficient is computed by a wake flow function.

In the conventional formulation, the dividing point of inner and outer layers is determined by the condition that the eddy viscosity coefficient of the inner layer matches those of the outer layer. However, the location of the dividing point so determined is too close to the wall compared with those given in Table 1. This is exactly the main reason for the inaccurate mean velocity profile given by BL model when applied to super/hypersonic turbulent boundary layers.

As stated above, the location of the dividing point of the inner and outer layers can be determined by using eq. (1), but then the wake flow function cannot be used in the outer layer. Otherwise the eddy viscosity coefficient will not be continuous at the dividing point. In fact, the variation of mixing length in the outer layer is not large, owing to the fact that the influence of the wall is not appreciable there. The implication is that there is no dominant mechanism of momentum transfer as in the inner layer, where the coherent structures dominate. Hence in the outer layer, we can set the mixing length to be a constant $l_{\text {mix } 0}$, namely

$$
l_{\text {mix }}=l_{\text {mix } 0} \text {. }
$$

This idea is not totally new, already mentioned elsewhere. Of course, there must be some error involved in this assumption, but as the gradient of the mean velocity in the outer layer is small, the total error in Reynolds stress there must also be small. To make the eddy viscosity coefficient continuous at the dividing point of the inner and outer layers, we should compute $l_{\text {mix } 0}$ by eq. (3) at the dividing point, namely $l_{\text {mix } 0}=\kappa y_{c}\left[1-\exp \left(-y_{c}^{+} / A^{+}\right)\right]$, where $y_{c}$ is the normal distance of the diving point from the wall.

Therefore, the new formulation is that the eddy viscosity coefficient in both inner and outer layers is determined by mixing the length theory as in eq. (2), but in the inner layer, the mixing length is determined by eq. (3), while in the outer layer, it is determined by eq. (4); and the dividing point is determined by eq. (1).

\subsection{Modification II: near wall modification for wall temperature condition}

Besides the Mach number and Reynolds number, the wall 
temperature condition is another factor affecting the characteristics of the compressible turbulent flows in the near wall region, especially for the hypersonic flows. As can be seen from the test cases below, for super/hypersonic turbulent boundary layers with adiabatic wall, Modification I alone is enough to yield satisfactory results. However, for isothermal cases, especially for the cold wall cases, Modification I alone is not enough, and a certain error still remains. While for most real flight cases, the wall temperature is lower than the corresponding adiabatic temperature, hence further modification is necessary.

As mentioned before, Duan et al.'s DNS [12] indicates that the effect of wall temperature condition manifests itself in the change of the thickness of the Sublayer in Figure 2. As the wall temperature goes down, the thickness of the Sublayer increases, implying that the region in which the Reynolds stress can be neglected compared to the viscous stress is enlarged, or equivalently, the eddy viscosity coefficient decreases in the near wall region, so is the mixing length determined by eq. (2).

Hence the modification can be done by modifying the constant $A^{+}$in eq. (3). The principle is, for adiabatic wall, $A^{+}$ keeps almost the original value 26 ; for cold wall, $A^{+}$should be larger than 26 , and the lower the wall temperature is, the larger $A^{+}$should be. From the DNs results, it is found that $A^{+}$can be expressed as:

$$
A^{+}=26\left(\frac{T_{0}}{T_{\mathrm{w}}}\right)^{0.6}
$$

where, $T_{0}=1+(\gamma-1) M^{2} / 2$, representing the total temperature of the oncoming flow, which is usually close to the adiabatic wall temperature, $\gamma$ is the specific heat ratio, and $T_{\mathrm{w}}$, the given wall temperature.

Since $A^{+}$appears in exponential function in eq. (3), and the dividing point of inner and outer layers is located nearly at the place $y^{+}=100$, the effect of the modification on $A^{+}$is mostly manifested only in the near wall region.

\subsection{Modification III: modification on the Prandtl number for aerothermal computations}

Modifications I and II are both for the modification of the eddy viscosity coefficient, resulting in the improvement of the mean velocity profile. However, for super/hypersonic boundary layers, aerothermal parameters are also important. Dong and Zhou [10] proposed a method for the modification of turbulent Prandtl number, which did improve the accuracy for the computation of aerothemal parameters.

The proposed modification on the turbulent Prandtl number is to let the eddy heat conductivity be expressed as

$$
k_{T}=\frac{1}{\max \left[\xi\left(k_{t} / k_{t, \max }\right), 1\right]} \frac{c_{p}}{0.9} \mu_{T},
$$

where $c_{p}$ is the specific heat at constant pressure, $k_{t} / k_{t, \max }=1-\left|1-15\left(1-\exp \left(-y^{+} / 7\right)\right) / y^{+}\right|^{1.8}$ is the normalized turbulent kinetic energy, the parameter $\zeta=\left(T_{0}-T_{\mathrm{w}}\right) / b+1$, and $b$ is an adjustable parameter, equal to 3.9 as suggested by then.

When the above modification was proposed, there were no modifications on the eddy viscosity coefficient. The result was, in spite of the improved accuracy of thermal parameters, the mean velocity profile and the wall friction coefficient remained inaccurate. Now, in this paper, modifications on the eddy viscosity coefficient are proposed, so $\mu_{T}$ in eq. (6) is modified. Consequently, parameter $b$ has to be readjusted. According to the DNS data referred in this paper, $b$ should be changed to be 8.0 , while the form of eq. (6) keeps unchanged.

Notice that the above modification is aimed at cold wall. For adiabatic and hot walls, $\xi \leqslant 1$, so $k_{T}=c_{p} \mu_{T} / 0.9$, remains the form for constant-turbulent-Prandtl-number, which is set to be 0.9 .

\section{Test cases}

In the following, several test cases are shown, in which the results of applying the BL model to super/hypersonic turbulent boundary layers both with and without the above modifications are compared with the DNS data, so to verify the validity of the modification.

The normal mesh size for BL model is the same as for DNS, so to avoid the effect of mesh size. The stream-wise grid spacing is 10-20 times of those for DNS.

The inlet condition for the BL model is derived from DNS data. The wall and upper bound conditions are the same as for DNS, and the extrapolation method is used as the outflow condition.

The high accuracy schemes are used, namely, the 5th order upwind compact difference scheme is used for the nonlinear term, the 6th order center compact difference scheme is used for the viscous term, and the 2nd order Runge-Kutta method is used for time advancing.

\subsection{Adiabatic turbulent boundary layer}

For the case of adiabatic wall, Modifications II and III have very little effect on the result of computation, but Modification I does have an appreciable effect.

The comparisons for Cases 1,3,5,6,10,11 and 14 of Table 1 are shown in Figure 5 to Figure 11 where the curves marked 'DNS' are DNS results, curves marked 'BL' are results computed by the original $\mathrm{BL}$ model, and curves marked 'modify' are results computed by the model with only Modification I. The cases mentioned above are all adiabatic cases except Case 3, whose wall temperature is only a little higher than the adiabatic wall temperature, and its 
results are almost not influenced by Modifications II and III. Therefore, these test cases can be used to verify Modification I. In addition, Case 3 is a boundary layer flow along a compression corner. Those shown in the figure correspond to the flow before the recirculation zone at the corner, so it is essentially a boundary layer flow on a flat plate.

The normal coordinates are all measured in terms of the nominal boundary layer thickness $\delta$, and $\mu_{T}$ is the eddy viscosity coefficient. It can be seen that the dividing point of inner and outer layers computed by the original BL model is too close to the wall compared with the DNS results, while the results computed by the modified model are very close

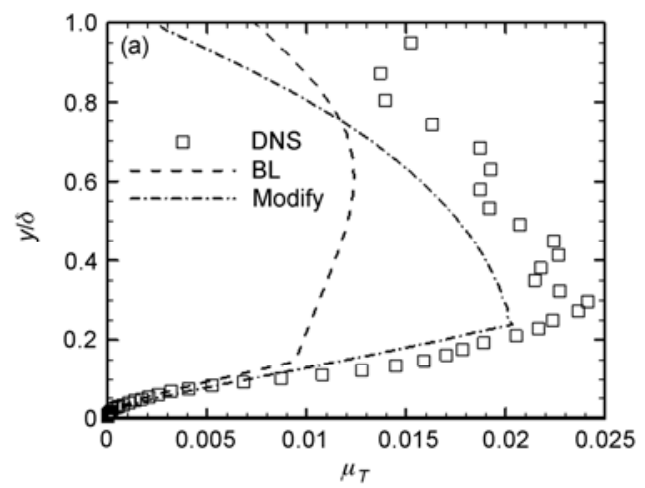

to the DNS results. And the mean velocity profile, mean temperature profile, as well as the skin friction coefficient are all greatly improved by the modification.

The conclusion is that for super/hypersonic turbulent boundary layers with adiabatic wall, modification I alone is sufficient to improve the result both on the mean velocity and temperature.

\subsection{Isothermal turbulent boundary layer}

Now consider cases (15-17) in which the wall temperature is different from the adiabatic wall temperature. Similar

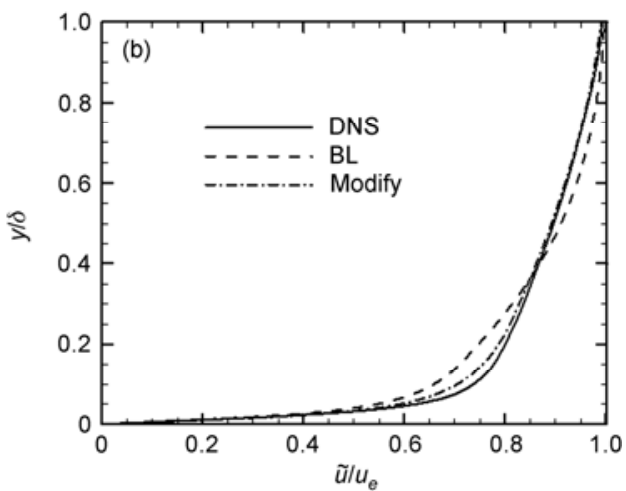

Figure 5 Case 1: Mach 2.5 turbulent boundary layer on a cone with adiabatic wall. (a) Eddy viscosity coefficient; (b) mean stream-wise velocity.
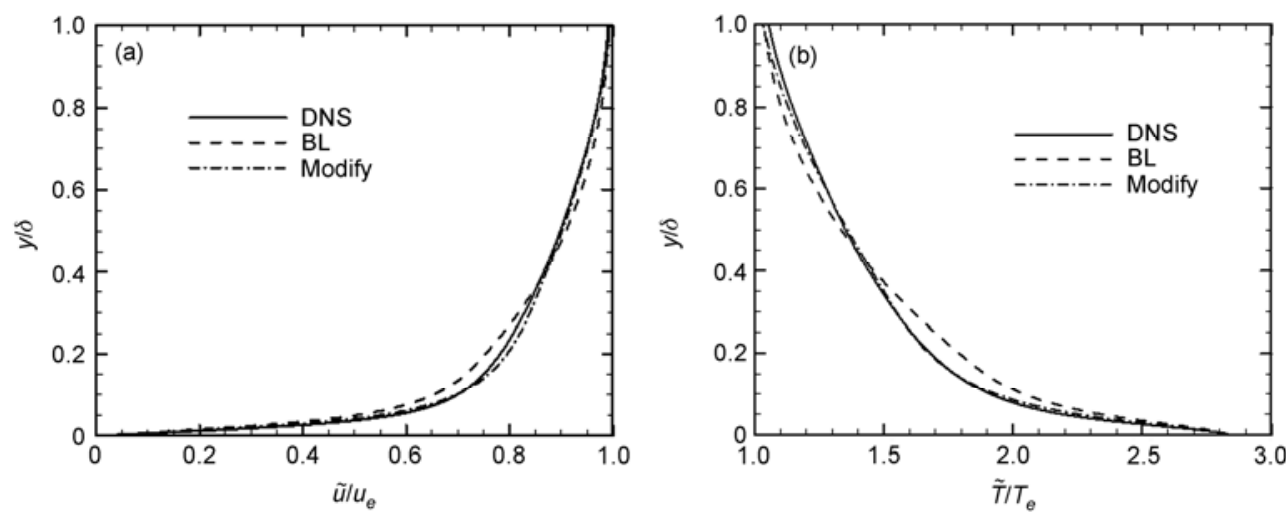

Figure 6 Case 3: Mach 2.9 turbulent boundary layer on a flat plate with nearly adiabatic wall. (a) Mean stream-wise velocity; (b) mean temperature.
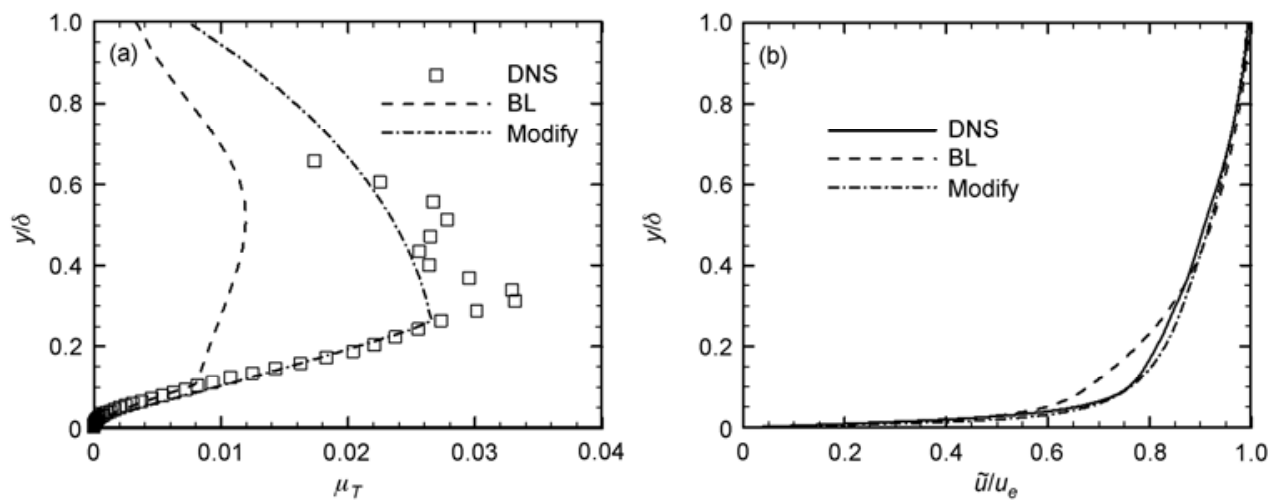

Figure 7 Case 5: Mach 3.5 turbulent boundary layer on a cone with adiabatic wall. (a) Eddy viscosity coefficient; (b) mean stream-wise velocity. 

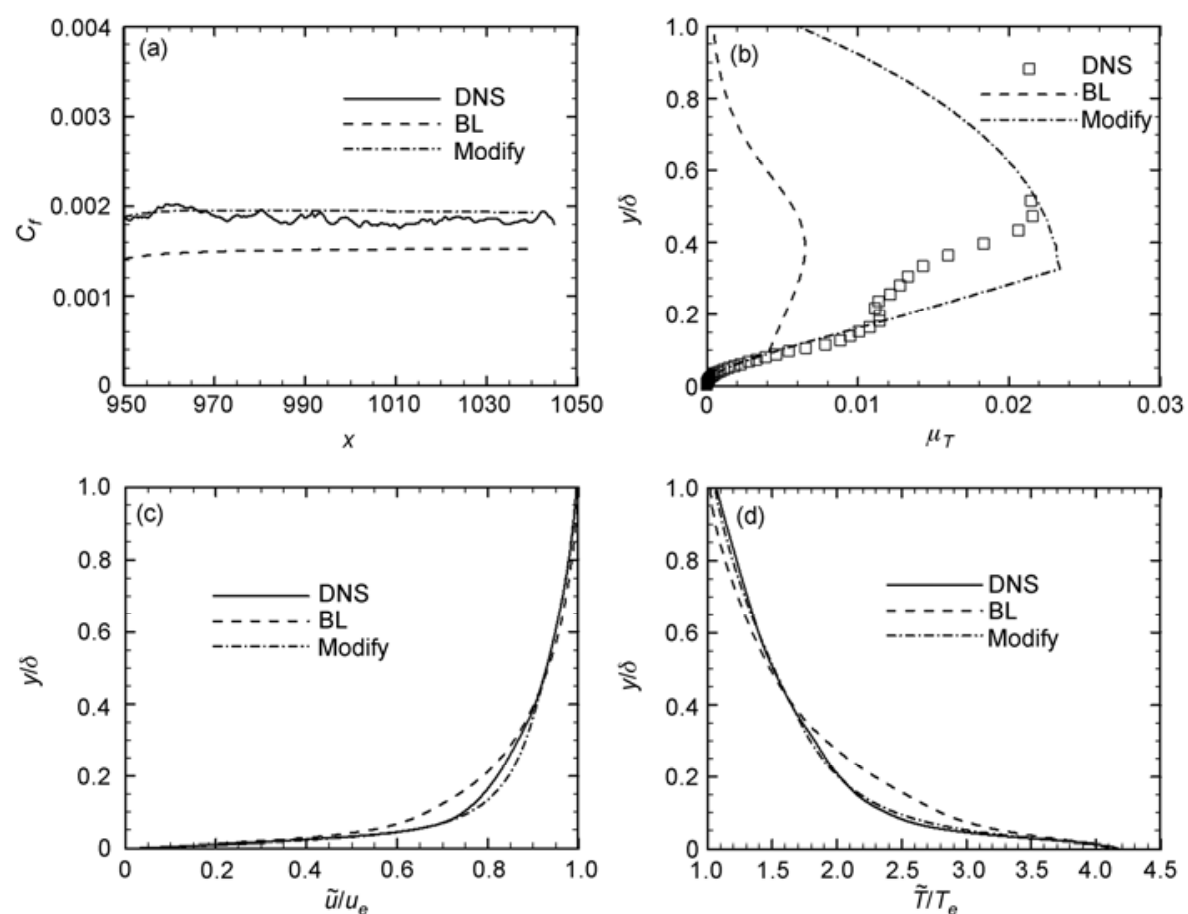

Figure 8 Case 6: Mach 4.5 turbulent boundary layer on a cone with adiabatic wall. (a) Skin friction coefficient; (b) eddy viscosity coefficient; (c) mean stream-wise velocity; (d) mean temperature.
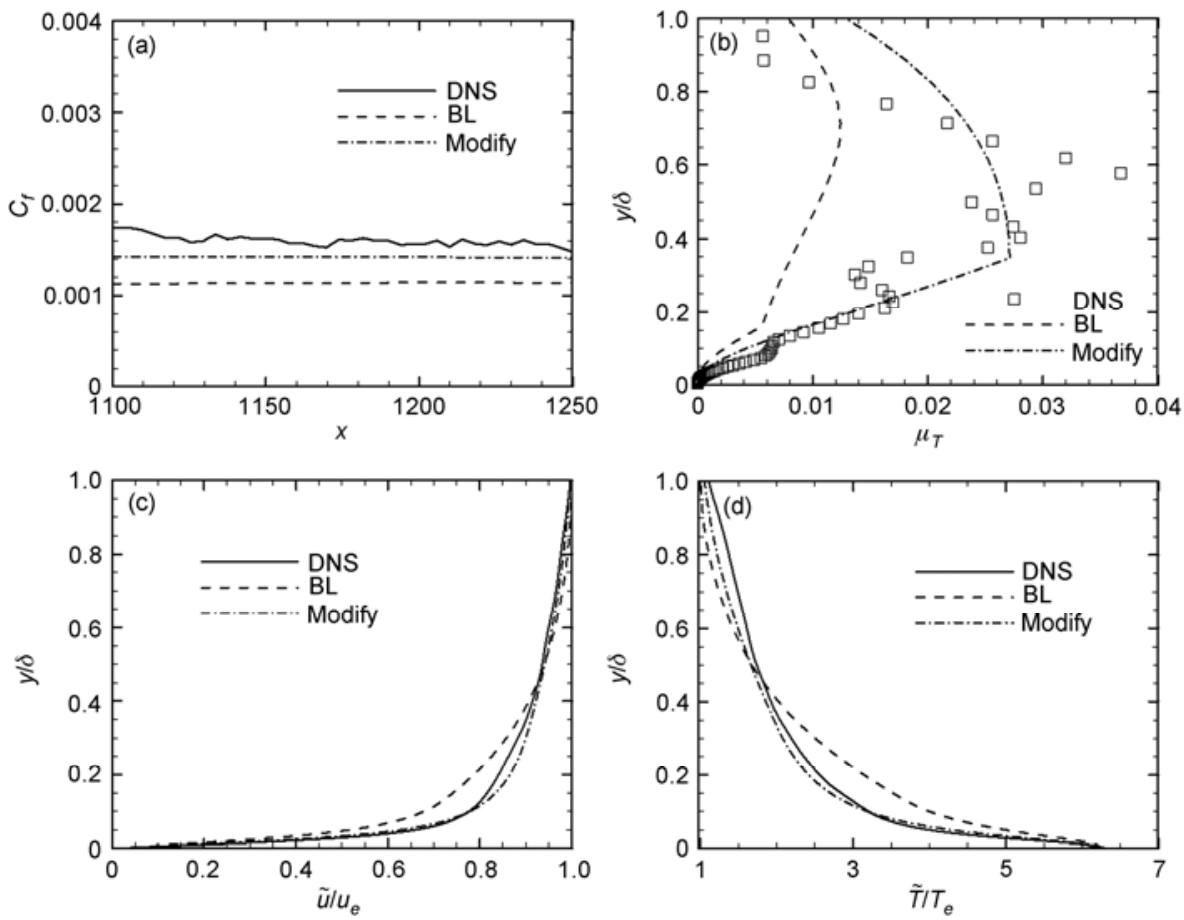

Figure 9 Case 10: Mach 6 turbulent boundary layer on a cone with adiabatic wall. (a) Skin friction coefficient; (b) eddy viscosity coefficient; (c) mean stream-wise velocity; (d) mean temperature.

comparisons are shown from Figure 12 to Figure 14. The oncoming Mach numbers are all 6, and the wall temperatures are 5.5, 4.5 and 3.72 times of the oncoming temperature respectively, or $0.74,0.61$ and 0.50 times of the adia- batic temperature, respectively.

Curves labeled with DNS are results from DNS, those labeled with $\mathrm{BL}$ are results from the original BL model, those labeled with modify 1 are results of BL model with 

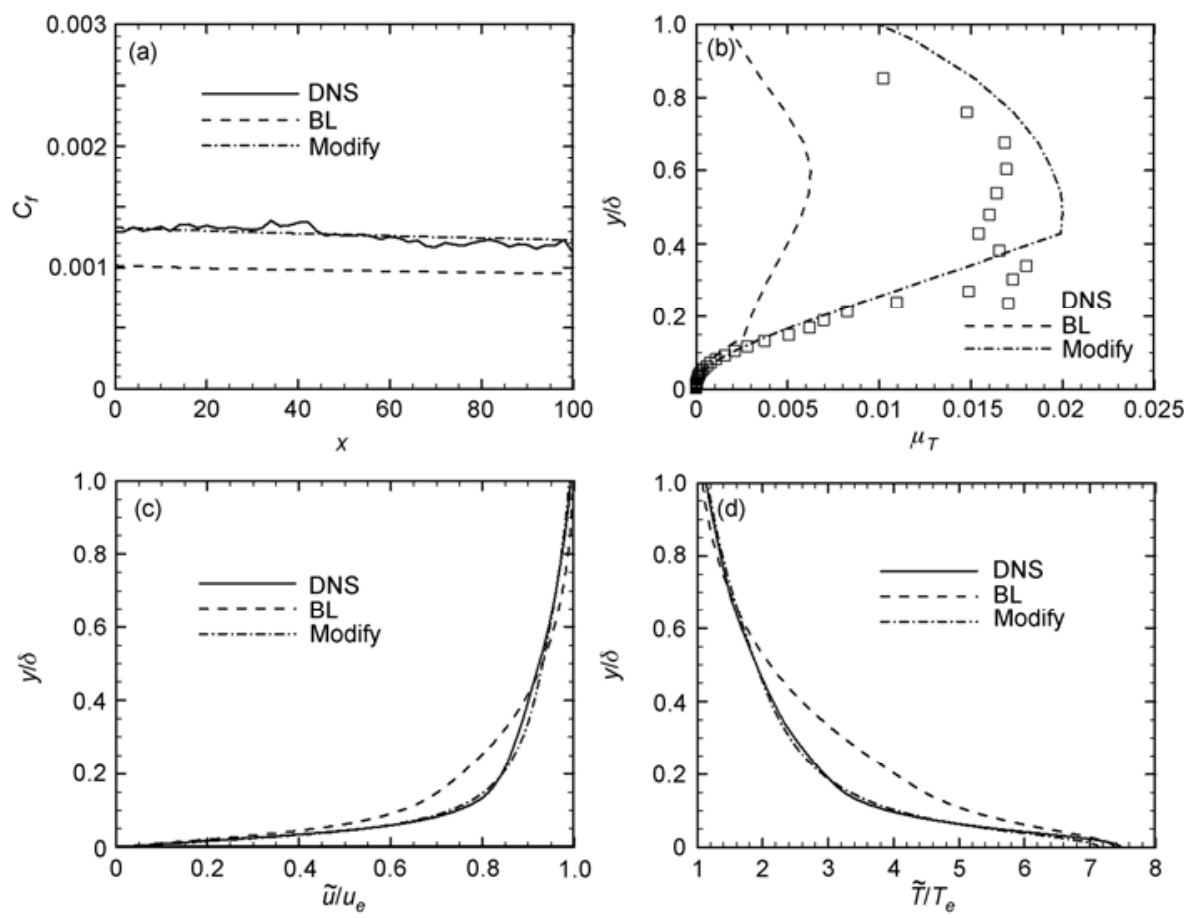

Figure 10 Case 11: Mach 6 turbulent boundary layer on a flat plate with adiabatic wall. (a) Skin friction coefficient; (b) eddy viscosity coefficient; (c) mean stream-wise velocity; (d) mean temperature.
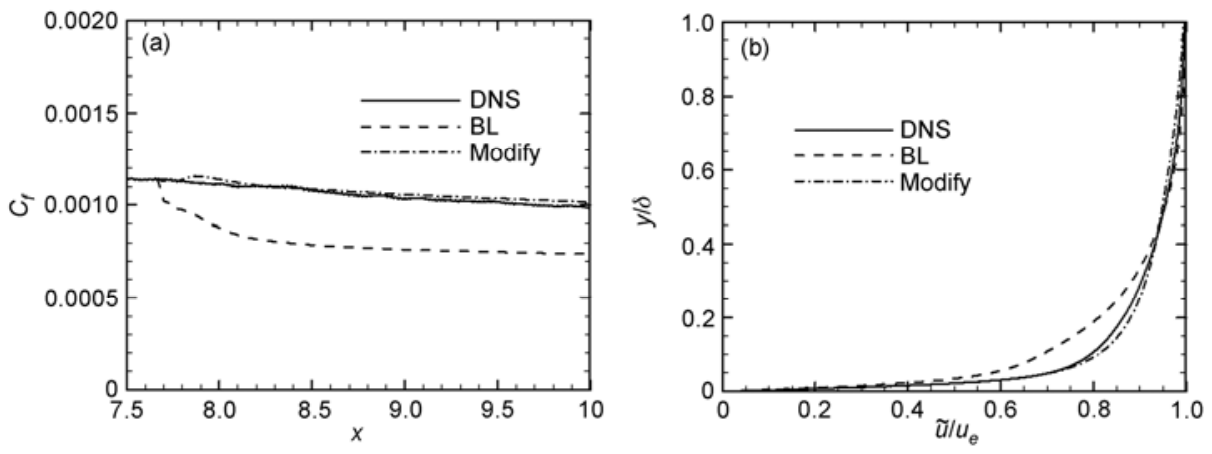

Figure 11 Case 14: Mach 6 turbulent boundary layer on a flat plate with adiabatic wall. (a) Skin friction coefficient; (b) mean stream-wise velocity.

only modification I, and those labeled with modify 2 are results from BL model with both modifications I and II, while those labeled with modify 3 are results from BL model with all three modifications.

It can be seen that the original BL model yields neither correct skin friction coefficient $C_{f}$ nor the overall shape of the mean velocity profiles. With modification I, the overall shape of the mean velocity profile becomes much closer to the result from DNS, but the skin friction coefficient does not. Then with near wall Modification II, the latter is also greatly improved. Modification III is mainly for heat parameters, so with little effect on the result of mean velocity profile and skin friction coefficient.

For thermal parameters, the original BL model yields neither correct heat flux at the wall nor the overall mean temperature profile. With Modification I, the overall mean temperature profile seems to have been greatly improved, but in the near wall region, it is not so, in that the heat flux $q_{w}$ does not agree with the DNS result. Adding Modification II does not solve this problem either; only with all the three modifications, the results become satisfactory. From the smaller figures in the upper parts of (e) and (f) in Figures $12-14$, one can clearly see the effect of different modifications in the near wall region, manifested in the improvement on the distributions of eddy viscosity coefficient and eddy heat conductivity coefficient in the near wall region, hence the improvement on the skin friction coefficient and heat flux at the wall.

\section{Conclusions}

(1) For $k-\varepsilon$ and $k$ - $\omega$ models, the expressions for the eddy viscosity coefficient, based purely on dimensional analysis, 

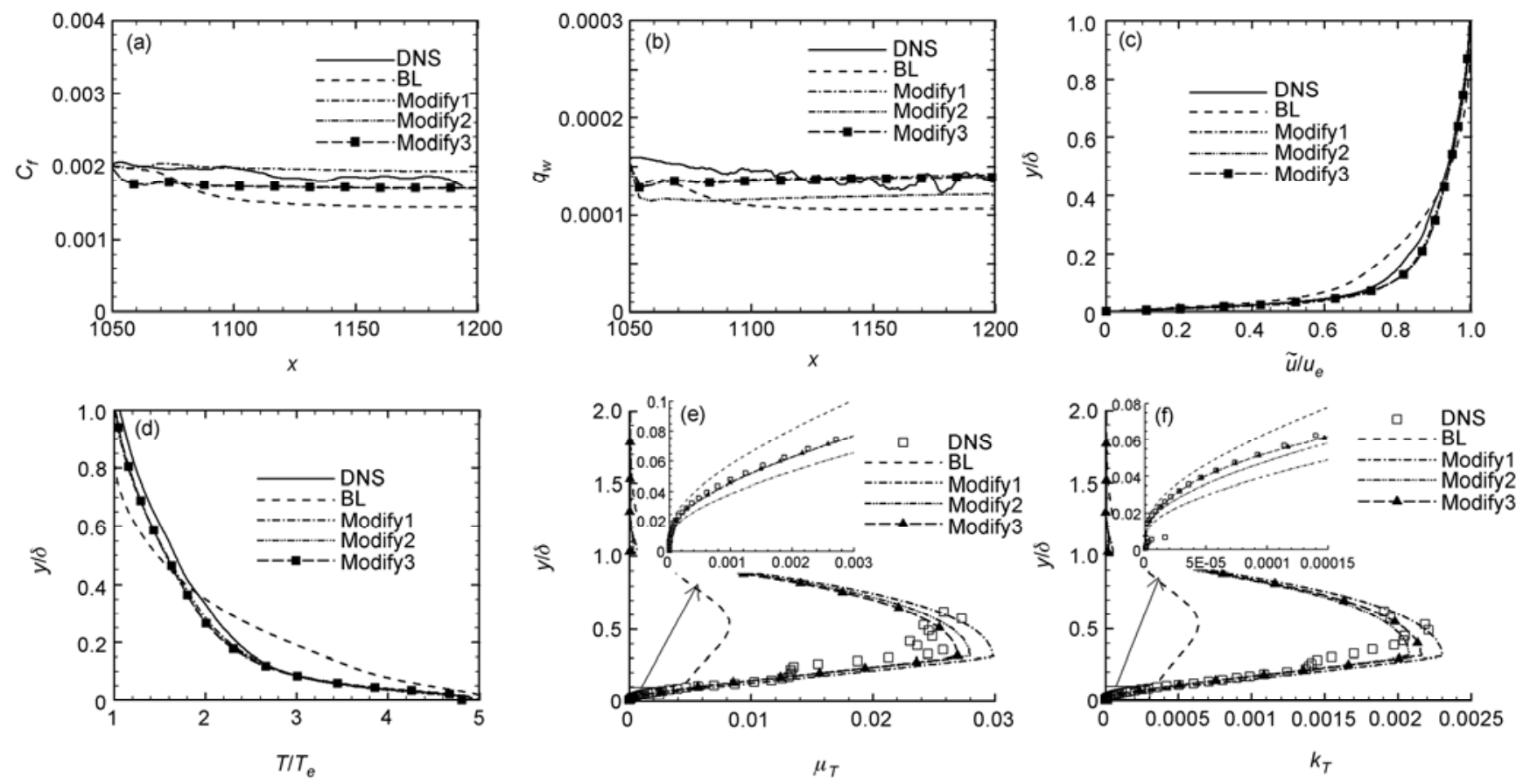

Figure 12 Case 15: turbulent boundary layer on a cone with Mach 6 and $T_{\mathrm{w}} / T_{\mathrm{aw}}=0.74$. (a) Skin friction coefficient; (b) skin heat flux; (c) mean stream-wise velocity; (d) mean temperature; (e) eddy viscosity coefficient; (f) eddy heat conductivity.
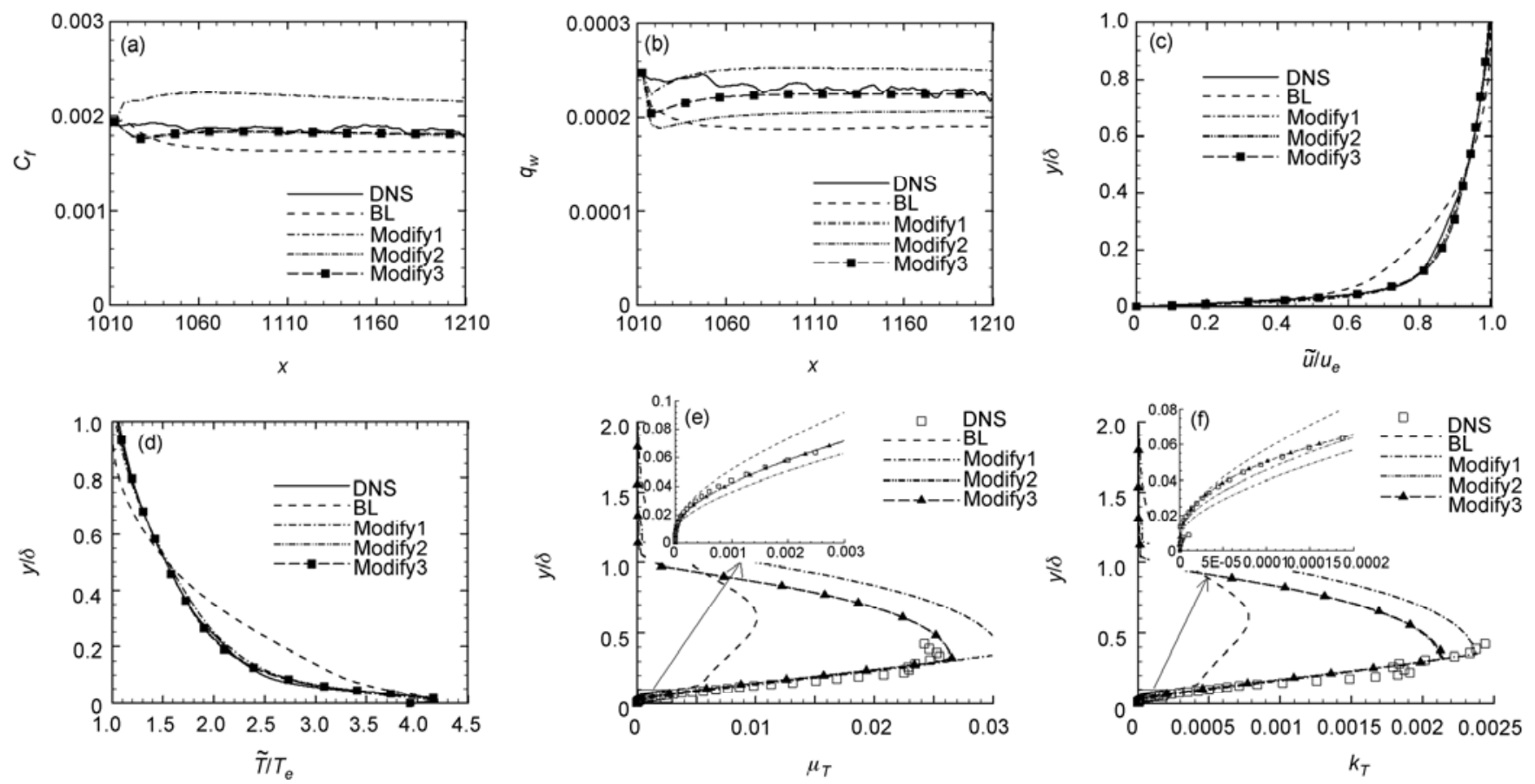

Figure 13 Case 16: turbulent boundary layer on a cone with Mach 6 and $T_{\mathrm{w}} / T_{\mathrm{aw}}=0.61$. (a) Skin friction coefficient; (b) skin heat flux; (c) mean stream-wise velocity; (d) mean temperature; (e) eddy viscosity coefficient; (f) eddy heat conductivity.

are incorrect. Further improvement is hard, except adding more adjustable parameters.

(2) For super/hypersonic turbulent boundary layers, the mean velocity profile and skin friction coefficients obtained by applying the BL model are inaccurate. The main problem manifests itself in that the location of the dividing point of inner and outer layers of the boundary layer, determined by the condition set in the BL model, is too close to the wall. In this paper, an empirical formula for determining the location of that dividing point has been proposed, based on re- 

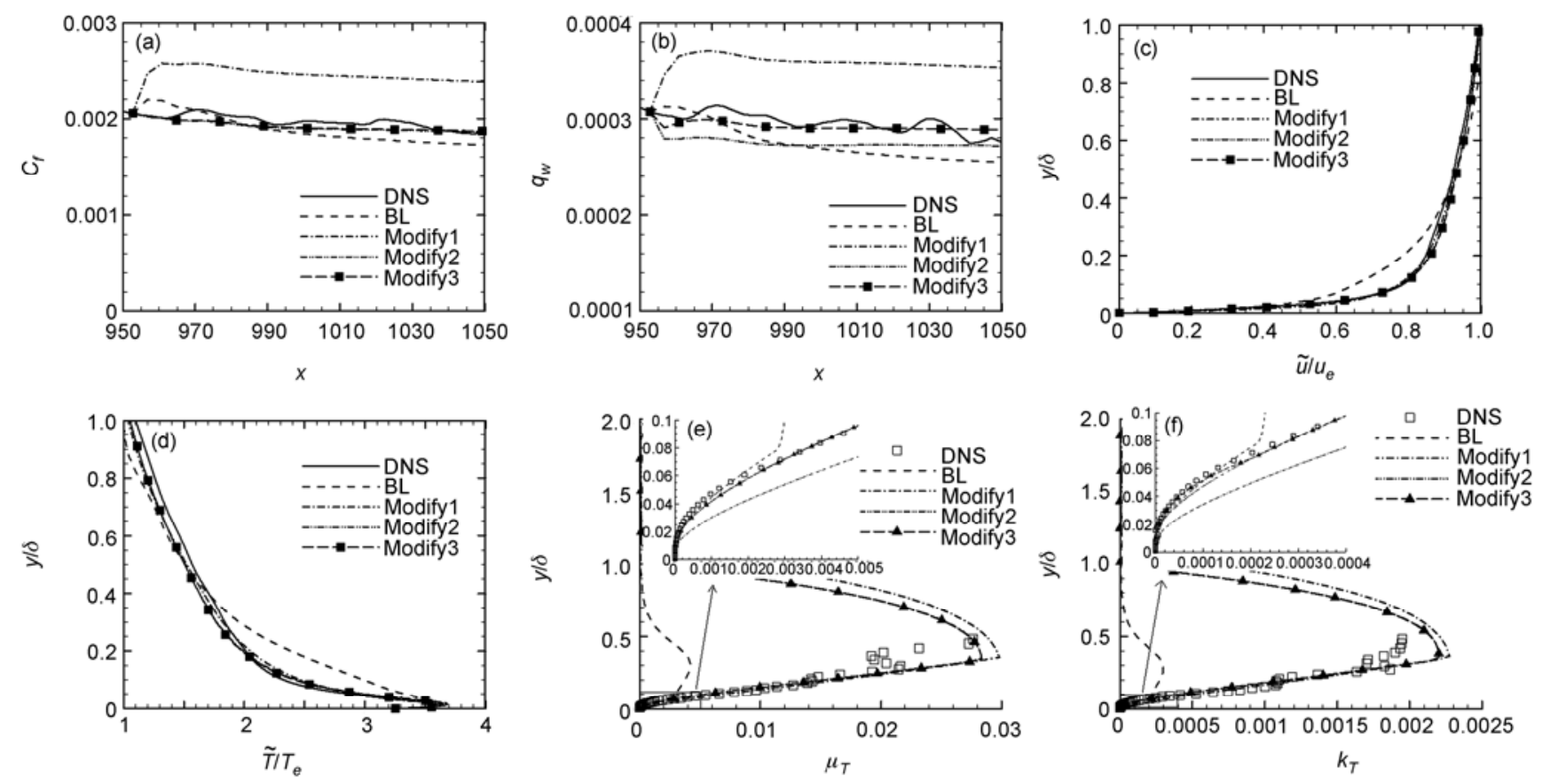

Figure 14 Case 17: turbulent boundary layer on a cone with Mach 6 and $T_{\mathrm{w}} / T_{\mathrm{aw}}=0.50$. (a) Skin friction coefficient; (b) skin heat flux; (c) mean stream-wise velocity; (d) mean temperature; (e) eddy viscosity coefficient; (f) eddy heat conductivity.

sults of a set of DNS for super/hypersonic turbulent boundary layers. A new method for the determination of the eddy viscosity coefficient in the outer layer of the boundary layer is correspondingly proposed.

(3) For super/hypersonic turbulent boundary layers with cold wall, the method of modifying the eddy viscosity coefficient in the near wall region is investigated. Also, a slight modification on the method of modifying the turbulent Prandtl number proposed in ref. [10], is proposed.

(4) The effectiveness of the modifications is tested by comparison with the results obtained by DND, including 7 cases with adiabatic or nearly adiabatic wall, and 3 cases with isothermal wall conditions. The result is satisfactory.

The cooperation of the two authors of this paper was brought about by Professor Zhou Heng of Tianjin University, and Professor Zhou has also made valuable contributions to the paper in the form of offering suggestions and comments to the first author, to which we are grateful. This work was supported by the National Basic Research Program of China (Grant No. 2009CB724103) and the National Aeronautics Base Science Foundation of China (Grant No. 2010ZA48002).

1 Baldwin B S, Lomax H. Thin layer approximation and algebraic model for separated turbulent flows. AIAA Paper, 1978, AIAA-78257

2 Spalart P R, Allmaras S R. A one equation turbulence model for aerodynamic flows. AIAA Paper, 1992, AIAA-92-0439

3 Menter F R. Two-equation eddy viscosity turbulence models for engineering applications. AIAA J, 1994, 32: 1598-1605

4 Chien K Y. Predictions of channel and boundary layer flows with a low Reynolds number turbulence model. AIAA J, 1982, 20: 33-38

5 Wilcox D C. Reassessment of the scale determining equation for advanced turbulence models. AIAA J, 1988, 26: 1299-1310
6 Roy C J, Blottner F G. Review and assessment of turbulence models for hypersonic flows. Prog Aerosp Sci, 2006, 42: 469-530

7 Wilcox D C. Turbulence Modeling for CFD. DCW Industries, 1994. $1-138$

8 Li X L, Fu D X, Ma Y W, et al. Acoustic calculation for supersonic turbulent boundary layer flow. Chin Phys Lett, 2009, 26: 094701

9 Dong M, Zhou H. Examination of the application of BL model in supersonic turbulent boundary layers (in Chinese). Acta Aerodyn Sin, 2009, 27: 102-107

10 Dong $\mathrm{M}$, Zhou $\mathrm{H}$. The improvement of turbulence modeling for the aerothermal computation of hypersonic turbulent boundary layers. Sci China Phys Mech Astron, 2010, 53: 369-379

11 Escudier M P. The distribution of mixing-length in turbulent flows near walls. Heat Transfer Section Report TWF/TN/12, 1966

12 Duan L, Beekman I, Martin M P. Direct numerical simulation of hypersonic turbulent boundary layers. Part 2. Effect of wall temperature. J Fluid Mech, 2010, 655: 419-445

13 Pirozzoli S, Grasso F, Gatski T B. Direct numerical simulation and analysis of a spatially evolving supersonic turbulent boundary layer at $M=2.25$. Phys Fluids, 2004, 16: 530-545

14 Li X L, Fu D X, Ma Y W, et al. Direct numerical simulation of shock/turbulent boundary layer interaction in a supersonic compression ramp. Sci China Phys Mech Astron, 2010, 53: 1651-1658

15 Maeder T, Adams N A, Kleiser L. Direct simulation of turbulent supersonic boundary layers by an extended temporal approach. J Fluid Mech, 2001, 429: 187-216

16 Martin M P. Direct numerical simulation of hypersonic turbulent boundary layers. Part 1 . Initialization and comparison with experiments. J Fluid Mech, 2007, 570: 347-364

17 Dong $\mathrm{M}$, Zhou $\mathrm{H}$. The effect of high temperature induced variation of specific heat on the hypersonic turbulent boundary layer and its computation. Sci China Phys Mech Astron, 2010. 53: 2103-2112

18 Li X L, Fu D X, Ma Y W. Direct numerical simulation of a spatially evolving supersonic turbulent boundary layer at $\mathrm{Ma}=6$. Chin Phys Lett, 2006, 23: 1519-1522

19 Tsujimoto K, Miyake Y. Universal property of autonomous layer in near-wall turbulence. In:IUTAM Symposium on Geometry and Statistics of Turbulence. Netherlands: Kluwer Academic Publication, 2001. 385-390 